\title{
Near fatal puerperal fever due to Mycoplasma hominis
}

\author{
Matthew J. Young* and Rosamond A. Cox ${ }^{1}$
}

Departments of Medicine and ${ }^{1}$ Pathology, Kettering and District General Hospital, Rothwell Road, Kettering, Northants NN16 8UZ, UK.

\begin{abstract}
Summary: We describe a case of severe septicaemia caused by Mycoplasma hominis in a 23 year old patient following childbirth. She developed disseminated intravascular coagulation and acute respiratory distress syndrome which have not hitherto been described in association with septicaemia due to this organism. Investigation and treatment leading to full recovery is outlined.
\end{abstract}

\section{Introduction}

Mycoplasma hominis is a common inhabitant of the female genital tract and is a well recognized cause of post-partum fever. ${ }^{1}$

Typically patients are not severely ill. They have a low grade fever for up to $\mathbf{4 8}$ hours after delivery, recovering uneventfully without antibiotic treatment. $^{2}$ We have been unable to discover any previous reports of life-threatening sepsis with disseminated intravascular coagulation and adult respiratory distress syndrome due to this species of Mycoplasma.

\section{Case report}

A 23 year old patient, gravida 3 , para 2 , was delivered of a normal male infant after induction at term and a 4-hour labour. Her pregnancy had been normal apart from folic acid deficiency anaemia. The placenta was intact. Forty eight hours after delivery she was discharged from hospital but within 4 hours she became feverish with rigors, headache, abdominal pain, thirst and polyuria. Three days later, having failed to respond to amoxycillin prescribed for a presumed urinary tract infection, she was readmitted to hospital.

On admission she was dyspnoeic, feverish $\left(39.4^{\circ} \mathrm{C}\right)$ and hypotensive $(100 / 60 \mathrm{mmHg})$ with a rapid pulse ( 240 beats $/ \mathrm{min}$ ). Examination revealed pulmonary crepitations and hepatosplenomegaly. The uterus was palpable up to the umbilicus and was not well contracted. The cervix was widely dilated and slight uterine tenderness was present, more on the right side than the left. The lochia was not offensive. The blood count showed typical

Correspondence: R.A. Cox, B.M., B.Ch., M.R.C.Path. *Present address: Lodge Moor Hospital, Sheffield S10 $41 \mathrm{H}$, UK.

Accepted: 18 July 1989 changes of disseminated intravascular coagulation (DIC) with haemoglobin $7.0 \mathrm{~g} / \mathrm{dl}$, platelets 71 $\times 10^{9} / 1$, reticulocytes $23 \%$ and fragmented distorted red cells on the peripheral blood film. Subsequent coagulation studies were consistent with this diagnosis. The prothrombin time was prolonged to 20 seconds (control 14 seconds) and kaolin-cephalin clotting time to 133 seconds (control 30 seconds). Fibrin degradation products were $40 \mathrm{mg} / \mathrm{l}$. The white blood cell count was $7.3 \times 10^{9} / 1$ and a differential count showed $95 \%$ neutrophils with left shift. The C-reactive protein was $324 \mathrm{mg} / \mathrm{l}$; liver function tests revealed a cholestatic picture. Her $\mathrm{PaCO}_{2}$ was $2.7 \mathrm{kPa}, \mathrm{PaO}_{2} 5.6 \mathrm{kPa}$ on air and arterial $\mathrm{pH}$ 7.51. The chest $\mathrm{X}$-ray showed widespread opacity described as 'ground glass'.

A provisional diagnosis of septicaemia was made and after blood cultures and a high vaginal swab had been taken, treatment was commenced with a one gram bolus of methylprednisolone together with metronidazole, gentamicin and ampicillin. The DIC was treated with intravenous heparin and blood products. Despite $100 \%$ oxygen via a face mask, her oxygenation deteriorated to a $\mathrm{PaO}_{2}$ of $4.4 \mathrm{kPa}$ and ventilation was commenced 8 hours after admission. Right atrial and later pulmonary wedge pressure monitoring was used to monitor fluid balance and haemodynamics.

After 48 hours her coagulopathy and infection seemed to be controlled but she developed further pyrexia. Intravenous ciprofloxacin was added after further blood cultures had been taken. She had no further episodes of pyrexia and continued to improve. Throughout the illness her renal function remained good. There was some indication of a reduction in glomerular filtration rate on the second day of illness when the serum creatinine rose to $150 \mu \mathrm{mol} / 1$ (upper limit of normal range $124 \mu \mathrm{mol} / \mathrm{l})$, but this had returned to normal the following day.

After 8 days ventilation she was extubated when 
her chest was clinically and radiologically clear and her hepatosplenomegaly had improved. She received a total of 14 days' antibiotics and was discharged 16 days after admission with a $\mathrm{PaO}_{2}$ of $15 \mathrm{kPa}$ and haemoglobin $13.3 \mathrm{~g} / \mathrm{dl}$. Her son had remained well.

A urine specimen taken on the day of admission was sterile. A high vaginal swab yielded Bacteroides species and numerous pus cells were seen on Gram film. M. hominis was not isolated despite the use of a selective/enrichment medium. A slow-growing pleomorphic Gram-negative bacillus was isolated from the anaerobic bottles of two blood cultures, one taken on the day of admission (when the patient was receiving amoxycillin) and one taken the day after admission (after commencement of gentamicin, metronidazole and ampicillin).

The bottles required 3 and 6 days' incubation respectively before the radiometric blood culture system gave a 'positive' reading. Subculture on to blood agar produced minute colonies visible with a hand lens after a further 3 days' incubation in anaerobic $/ \mathrm{CO}_{2}$ enriched atmosphere. Attempts to identify the organism using conventional laboratory techniques were unsuccessful and it was identified as $M$. hominis by the Mycoplasma Reference Facility of the National Collection of Type Cultures.

\section{Discussion}

The association between $M$. hominis and infections of the genital tract have been recognized for at least 30 years. ${ }^{3}$ It was first reported to cause post-partum septicaemia by Tulley and Smith in $1968 .{ }^{4}$ Since then a number of investigators have drawn attention to the importance of $M$. hominis in post abortal and post partum fever. ${ }^{1,2,5}$ Platt et al. ${ }^{1}$ followed up 535 patients after vaginal delivery and found that $M$. hominis septicaemia was the commonest cause of fever.

Almost all reports of $M$. hominis septicaemia following abortion or childbirth have emphasized the benign nature of the infection. Even a patient with severe immunosuppression secondary to malignant lymphoma, recovered from $M$. hominis septicaemia without antibiotic treatment effective against the infecting organism. ${ }^{6}$

DIC is a recognized complication of $M$. pneumoniae infections ${ }^{7}$ but has not been recorded in association with $M$. hominis septicaemia, or with other conditions such as pelvic inflammatory disease and pyelonephritis where $M$. hominis acts as a primary pathogen.

It is likely that the source of $M$. hominis in our patient was the genital tract. Studies of vaginal flora in asymptomatic premenopausal, non-pregnant women have shown that this organism was present in 12 to $22 \%$ of the women studied. ${ }^{8} \mathrm{We}$ failed to isolate $M$. hominis from a high vaginal swab taken on the day of admission. Possibly overgrowth of Bacteroides species obscured the tiny colonies of $M$. hominis on blood agar. No growth occurred on the selective medium we use to detect $M$. hominis in genital specimens. This medium contains thallous acetate at a concentration of $0.01 \%$ and did not support the growth of the blood culture isolates.

It is possible that our patient's acute respiratory failure was a direct result of $\boldsymbol{M}$. hominis pneumonia and that her pregnancy and childbirth were incidental to her illness. M. hominis can be isolated from the respiratory tract of up to $3 \%$ of healthy adults. ${ }^{9}$

However, unlike pneumonia due to $M$. pneumoniae, that due to $M$. hominis seems to be extremely rare in immunocompetent individuals, although cases have been reported in patients with profound immunosuppression. ${ }^{10}$

$M$. hominis is sensitive to tetracycline, but many strains are resistant to gentamicin and other antibiotics used for the treatment of septicaemia. Ciprofloxacin has been shown to be active in vitro against $M$. hominis, but evidence of its clinical efficacy is lacking. ${ }^{11}$ It is difficult to know whether it contributed to our patient's recovery.

We therefore feel that in patients with severe post partum septicaemia, $M$. hominis should be considered as a possible cause and antimicrobial treatment should be selected with this in mind.

\section{References}

1. Platt, R., Juey-Shin, L.L., Warren, J.W., Rosner, B., Edelin, K.C. \& McCormack, W.M. Infection with Mycoplasma hominis in post partum fever. Lancet 1980, ii: 1217-1221.

2. Taylor-Robinson, D. \& McCormack, W.M. The genital mycoplasmas. N Engl J Med 1980, 302: 1003-1010.

3. Russell, F.E. \& Fallon, R.J. Mycoplasma and the urogenital tract. Lancet 1970, i: 1295.

4. Tulley, J.G. \& Smith, L.G. Post partum septicaemia with Mycoplasma hominis. JAMA 1968, 204: 827-828.

5. Thomas, M.E.M. \& Andrews, B.E. Mycoplasma in urogenital tract. Lancet 1970, ii: 366-367.

6. Friis, H., Plesner, A., Scheibel, J., Justesen, T. \& Lind, K. Mycoplasma hominis septicaemia. Br Med J 1983, 286: 2013-2014.

7. Mulder, L.J.M.M. \& Spierings, E.L.H. Stroke due to intravascular coagulation in Mycoplasma pneumoniae infection. Lancet 1987, ii: 1152-1153.

8. Hill, G.B., Eschenbach, D.A. \& Holmes, K.K. Bacteriology of the vagina. In: Mardh, P.A. \& Taylor-Robinson, D. (eds) Bacterial Vaginosis. Almquist and Wiksell International, Stockholm, 1984, pp. 23-39. 
9. Mufson, M.A Mycoplasma hominis: a review of its role as a respiratory tract pathogen of humans. Sex Transm Dis 1983, 10 (Suppl.): 335-340.

10. Roberts, D., Murray, A.E., Pratt, B.C. \& Meigh, R.E. Mycoplasma hominis as a respiratory pathogen in X-linked hypogammaglobulinaemia. J Infect 1989, 18: 175-177.
11. Ridgeway, G.L., Muntaz, G., Gabriel, F.G. \& Oriel, J.D. The activity of ciprofloxacin and other 4 - quinolones against Chlamydia trachomatis and Mycoplasmas in vitro. Eur J Clin Microbiol 1984, 3: 344-346. 